\title{
Selection and preference for botulinum toxins in the management of photoaging and facial lines: patient and physician considerations
}

This article was published in the following Dove Press journal:

Patient Preference and Adherence

25 August 2010

Number of times this article has been viewed

\author{
Paul S Yamauchi ${ }^{1,2}$ \\ 'Dermatology Institute and Skin \\ Care Center, Santa Monica, \\ CA, ${ }^{2}$ Division of Dermatology, \\ David Geffen School of Medicine \\ at UCLA, Los Angeles, CA, USA
}

Correspondence: Paul S Yamauchi Dermatology Institute and Skin Care Center, 200I Santa Monica Blvd, Ste 490W, Santa Monica, CA 90404, USA $\mathrm{Tel}+\mathrm{I}(3 \mathrm{I}$ ) 829-4I04 Fax +I (3I0) 829-4I50 Email paulyamauchi@yahoo.com

\begin{abstract}
Various noninvasive to minimally invasive techniques can be used for the improvement of cutaneous changes seen with photoaging. These include dermabrasion, chemical peels, ablative and nonablative lasers, and filler agents such as hyaluronic acid. However, the most common nonsurgical cosmetic procedure performed in the treatment of rhytides is injection with botulinum toxin. Its extensive safety history and relative ease of use by the practitioner has led to high satisfaction in millions of patients. Nonetheless, proper training of the fundamentals in injection technique, the choice of the appropriate candidate, and knowledge of potential adverse events are imperative to ensure a satisfactory and safe outcome.
\end{abstract}

Keywords: botulinum toxin, rejuvenation, photoaging, rhytides

Dynamic facial lines can be interpreted as manifestations of negative emotions, fatigue, stress, and perceived aging. Such rhytides result as a combination of intrinsic skin aging, ultraviolet damage, and repetitive action of facial muscles. Of these, the most contributing force for the persistence of facial wrinkles is the repetitive contractions of the intrinsic muscles for facial expression. There are a variety of different treatment options available for facial lines including the use of filling agents such as hyaluronic acid, ablative and nonablative laser surgery, dermabrasion, chemical peels, and invasive surgery such as rhytidectomy. However, these procedures do not adequately address the underlying cause of dynamic wrinkles as evidenced by the persistence of facial lines after treatment. The understanding that muscular contraction contributes to etiology of facial lines, wrinkles, and furrows ${ }^{1}$ has broadened the treatment options for these facial cosmetic blemishes.

Botulinum toxin type A (BTX-A), a potent neurotoxin that irreversibly blocks presynaptic acetylcholine release, has been successfully employed to treat facial spastic conditions such as blepharospasm, strabismus, focal dystonias, spasmodic dysphonia, and achalasia. ${ }^{2}$ Consequently, many clinicians have noted improvement of facial rhytides in patients who received BTX-A for these spastic disorders. Indeed, while treating patients with blepharospasm, Carruthers and Carruthers first noted improvement of glabellar frown lines. ${ }^{3}$

A variety of other hyperkinetic facial lines such as crow's feet, horizontal forehead lines, melolabial folds, and other hyperkinetic facial lines have been successfully treated with BTX-A. ${ }^{4,5}$ Currently there are two commercial formulations of BTX-A, Botox ${ }^{\circledR}$ (Allergan, Inc.) and Dysport ${ }^{\mathrm{TM}}$ (Medicis) approved in the United States. In 2002, Botox ${ }^{\circledR}$ Cosmetic was FDA approved for the temporary improvement in the appearance of moderate to severe glabellar lines associated with corrugator and/or procerus muscle 
activity in adult patients aged 65 years or less. In 2009, Dysport was FDA approved for the same cosmetic indication as Botox Cosmetic. In addition, Bocouture ${ }^{\circledR} /$ Xeomin $^{\circledR}$ is another BTX-A formulation that is marketed by Merz Pharmaceuticals (Frankfurt, Germany) and was approved in late 2009 in Germany for the treatment of glabellar lines in adults younger than 65 years of age.

Likewise, botulinum toxin type B (BTX-B) is another potent neurotoxin that has been employed to treat spastic conditions such as cervical dystonia. Myobloc ${ }^{\circledR}$, the only commercial formulation of BTX-B, is manufactured by Solstice Neurosciences, Inc. and is FDA approved for the treatment of cervical dystonia but currently not approved in the United States for any cosmetic use. A variety of hyperkinetic facial lines have also been treated successfully with BTX-B. ${ }^{6-13}$

The focus of this review article is to investigate the pharmacology of botulinum toxins in the management of skin aging, efficacy studies pertaining to various types of botulinum toxins, the safety and tolerability of botulinum toxin, and physician and patient reported outcomes relating to satisfaction, preference, and convenience. Table 1 lists the highlights of the various approved botulinum toxins.

\section{Pharmacology - structure and mechanism of action}

There are seven serologically distinct types of botulinum neurotoxins, designated types A through $\mathrm{G}$, which correspond to the strain of Clostridium botulinum that produces the specific toxin. These serotypes are all synthesized as single chain polypeptides with molecular weights of approximately $150 \mathrm{kD}$ and share a common structural organization which consists of one heavy chain $(100 \mathrm{kD})$ and one light chain $(50 \mathrm{kD})$ polypeptide that are linked by a single disulfide bond. All botulinum toxins inhibit acetylcholine release at the neuromuscular junction in a three-step process. The single-chain polypeptides are activated by proteases which are cleaved into a double chain consisting of the heavy chain and the light chain moieties. ${ }^{14}$ Upon cleavage, the heavy chain binds to a high affinity receptor on the presynaptic nerve terminal which enables the internalization of the bound toxin into the cell. The light chain moiety is a zinc-dependant endopeptidase that cleaves membrane proteins responsible for docking acetylcholine vesicles on the inner side of the nerve terminal membrane. ${ }^{15}$ The cleavage of these proteins inhibits the fusion of the vesicles with the nerve membrane, thereby preventing release of acetylcholine into the neuromuscular junction. ${ }^{16}$

The intracellular target of BTX-A is the SNAP-25 (synaptosome-associated protein of molecular eight $25 \mathrm{kD}$ ) which is a protein essential for the successful docking and release of acetylcholine vesicles with the presynaptic vesicle. ${ }^{17}$ In contrast, BTX-B cleaves VAMP (vesicle associated membrane protein), which is also known as synaptoabrevin. ${ }^{18}$ Like SNAP-25, VAMP is essential for the docking and fusion of the synaptic vesicles to the presynaptic membrane for the release of acetylcholine.

\section{Cosmetic uses of botulinum toxin Botox Cosmetic}

Botox Cosmetic manufactured by Allergan Inc. was the first Type A botulinum toxin to be approved by the FDA in the United States. The product is supplied as a vacuum-dried lyophilized power in vials containing either $50 \mathrm{U}$ or $100 \mathrm{U}$ of Botox Cosmetic. Unreconstituted Botox Cosmetic should be stored at $2-8^{\circ} \mathrm{C}$. The official recommendation by the manufacturer is to reconstitute Botox Cosmetic with $2.5 \mathrm{~mL}$ of $0.9 \%$ nonpreserved saline for the $100 \mathrm{U}$ vial $(1.25 \mathrm{~mL}$ for the $50 \mathrm{U}$ vial) to a final concentration of $4.0 \mathrm{U} / 0.1 \mathrm{~mL}$. Practitioners have also used other different dilutions ranging from 1 to $10 \mathrm{~mL} / 100 \mathrm{U}$ and have reported that dilution at higher volumes can result in more diffusion. In addition, researchers have demonstrated no decrease in efficacy using preserved saline to dilute the Botox Cosmetic, although there is less pain experienced upon injection using preserved saline. ${ }^{19}$ The manufacturer recommends avoiding aggressive agitation of the vial upon mixing to prevent denaturation of the product. The reconstituted product should never be frozen and can be stored for up to six weeks at $4{ }^{\circ} \mathrm{C}$.

Table I Highlights of the various botulinum toxins

\begin{tabular}{lllll}
\hline & Botox & Dysport & Myobloc & Bocouture/Xeomin \\
\hline Type of botox & A & A & B & A \\
Manufacturer & Allergan & Medicis & Solstice neuroscience & Merz \\
FDA cosmetic approval & Glabellar lines & Glabellar lines & None & None (Approved In Germany for glabellar lines) \\
Other indications & $\bullet$ Cervical dystonia & $\bullet$ Cervical dystonia & $\bullet$ Cervical dystonia & $\bullet$ Cervical dystonia \\
& $\bullet$ Blepharospasm & & & $\bullet$ Blepharospasm \\
& $\bullet$ Strabismus & & & \\
& $\bullet$ Axillary hyperhidrosis & & & \\
\hline
\end{tabular}


Tuberculin or insulin syringes are frequently used for injecting Botox Cosmetic since there is no potential space in the hub and result in less waste of the product. Either 30- and 32-gauge needles are the most commonly used for injecting Botox Cosmetic. ${ }^{20}$ Topical anesthesia is not required but some patients may request this to minimize discomfort. The use of ice can also reduce pain and also minimize purpura formation by constricting the blood vessels prior to injection.

Prior to treating the patient with Botox Cosmetic, a complete medical history should be attained to prevent unwanted reactions. ${ }^{21}$ Medications and food products that inhibit platelet aggregation and increase clotting time should be discontinued for 10-14 days prior to the procedure. These include aspirin, nonsteroidal anti-inflammatories, garlic, and fish oils. Some medical conditions that are contraindicated for botulinum toxin injections include peripheral motor neuropathic diseases or neuromuscular functional disorders, such as myasthenia gravis and Eaton-Lambert Syndrome. Patients who are currently taking aminoglycoside antibiotics such as gentamycin may develop increased inhibition of neuromuscular transmission and concomitant usage together with botulinum toxin is contraindicated. The presence of any infection or inflammation on the skin at the site of injection is also a contraindication. Finally, females who are pregnant or lactating should not receive injections of botulinum toxin.

\section{The upper face}

Facial wrinkles involving the forehead, glabella, and lateral periorbital regions are common aesthetic areas where botulinum toxin is injected. In treating the glabella, botulinum toxin targets thee bilateral corrugators, depressor supercilii, and the central procerus muscles that are responsible for the glabellar lines. Usually, two injections are administered per corrugator muscle and one injection in the procerus. The first phase 3 study demonstrating the efficacy of Botox Cosmetic for the treatment of glabellar lines was published in 2002. ${ }^{22}$ Patients with moderate to severe glabellar lines at maximum frown received intramuscular injections of $20 \mathrm{U}$ Botox Cosmetic or placebo into 5 glabellar sites. Patients were followed up for 120 days after injection. Outcome measures were physician ratings of glabellar line severity at maximum frown and rest, patient assessment of improvement, and vital sign and adverse event monitoring. A total of 264 patients were enrolled, with 203 receiving Botox Cosmetic and 61 in the placebo group. There was a significantly greater reduction in glabellar line severity with BTX-A than with placebo in all measures $(P<0.022)$ and the effect was maintained for many patients through to day 120 . The recommended dosage for the treatment of glabellar lines is $20 \mathrm{U}$ but can vary from 15-30 U depending on the strength of the corrugator and procerus muscles. Typically, men may need higher doses of botulinum toxin versus women given the greater strength of the muscles.

The treatment of the glabellar region with botulinum toxin results in a slight brow lift by weakening the central frontalis effect of brow elevation and thus proportionately increasing the frontalis effect of lateral brow elevation. ${ }^{23} \mathrm{An}$ advanced technique that can be utilized to attain a lateral brow lift by weakening the lateral lid depressor muscles is to inject 1-2 $\mathrm{U}$ of Botox Cosmetic into the lateral-superior orbicularis muscle just below the lateral brow but above the superior orbital rim.

A single-center, prospective, double-blind, randomized, dose-comparison study was conducted in female patients between the ages of 18 and 65 years of age for the treatment of glabellar, forehead, and periorbital rhytides with Botox Cosmetic. ${ }^{5}$ Eligible patients were assigned randomly to 1 of 3 treatment groups representing total doses of 32,64 , or $96 \mathrm{U}$ and were followed up for up to 52 weeks. Patients were required to have upper facial wrinkles that met the following specifications on a 4 -point facial wrinkle scale $(\mathrm{FWS})(0=$ none; $1=$ mild; $2=$ moderate and $3=$ severe as determined at maximum attempted muscle contraction by a trained observer); moderate or severe glabellar lines; mild, moderate, or severe forehead lines; and bilaterally symmetric moderate or severe crow's feet. The total dose was divided evenly among 16 injection sites: 5 injections in the glabellar, 5 injections in the forehead, and 3 injections in each lateral canthal area for the periorbital wrinkles. Botox Cosmetic treatment, used in total doses of 32,64 , or 96 $\mathrm{U}$, was shown to be safe and effective for treating multiple upper facial rhytides in female patients in single sessions. Overall, each dose resulted in significant improvements in the appearance of facial rhytides, but a dose-response relationship emerged based on duration of effect and on between-group differences on a substantial number of the measures that were assessed. The principal differences between the groups were in the duration of the effect, although in some individuals, some areas wore off more rapidly than other areas. Based on anecdotal information, some physicians hypothesize that a longer duration of rhytide effacement may be achieved through treatment of multiple areas, as this eliminates any potential for adjacent muscle recruitment. 
When using botulinum toxin to treat forehead rhytides, typical doses of 15-30 U of Botox Cosmetic are injected into the frontalis muscle. ${ }^{24}$ Because the forehead is the primary brow elevator, care and caution must be exercised not to lower the eyebrows and flatten them when treating forehead wrinkles, especially in women where an arched brow is of aesthetic importance. To avoid lowering the brow, injecting higher up in the forehead can minimize this tendency as well as treating the glabella concomitantly. When treating the forehead for the first time, practitioners may reserve treating of the forehead area for 2 weeks after injecting the glabellar area first and also utilize a lower dose for the forehead to reduce the chance of depressing the brow excessively.

Typically, three injections are performed on each side of the lateral orbital of the eye to target the lateral orbicularis muscle to treat crow's feet, with 2-3 U of Botox Cosmetic used per injection..$^{25}$ The distance from the lateral canthus to the area of injection should be around $1-1.5 \mathrm{~cm}$ to minimize the rare occurrence of diplopia. In addition, the area around the lateral orbit is enriched with blood vessels and this is a site more prone to purpura. Care should be exercised to closely examine for the presence of blood vessels prior to injection. Sometimes after successful treatment of the crow's feet, patients may note increased crinkling and fine rhytide formation immediately below the infraorbital area due to muscle recruitment. Should this occur, $1 \mathrm{U}$ of Botox Cosmetic can be placed in the area of new wrinkle formation to obviate this effect. A study was conducted to compare the efficacy and safety of 4 doses of Botox Cosmetic with placebo to treat crow's feet. ${ }^{4}$ Participants received a single bilateral treatment of 18, 12, 6, or $3 \mathrm{U}$ of BTX-A or placebo injected into the lateral aspect of the orbicularis oculi muscle. Investigators and participants rated crow's feet severity at maximum smile on day 7 and at 30-day intervals from days 30 to 180 . As observed by both investigators and subjects, all doses of BTX-A resulted in improvements in crow's feet severity when compared with placebo. A dose-dependent treatment effect for efficacy was observed, with higher doses having an increased magnitude and duration of effect. However, a clear differentiation between the $18 \mathrm{U}$ and $12 \mathrm{U}$ doses was not apparent. It was concluded that BTX-A was safe and effective in decreasing the severity of crow's feet, with $12 \mathrm{U}$ per side suggested as the most appropriate dose.

\section{Mid and lower face}

The mid and lower face is not a common area to treat with botulinum toxin when compared to the upper face. Caution must be exercised in these areas since diffusion of toxin and inadvertent injection of adjacent musculature can result in functional deficits and unwanted limited motion.

Nasal wrinkles on the dorsum of the nose can frequently be treated with botulinum toxin by injecting a few units into the nasalis muscle. Between 2 and $5 \mathrm{U}$ of botulinum toxin have commonly been used. A study was conducted to determine the efficacy of Botox Cosmetic in the treatment of nasal wrinkles of the superior nose (bunny lines). ${ }^{26} \mathrm{~A}$ total of 250 patients with nasal rhytides were treated and $3 \mathrm{U}$ of Botox Cosmetic were injected bilaterally into the nasalis muscle. Patients were seen at 1 month for follow-up, and the remaining rhytides were documented. In total, $40 \%$ of patients had satisfactory treatment of nasal wrinkles with the initial bilateral 3-U injections. The remaining $60 \%$ still had nasal rhytides following the nasalis muscle injections. An additional $30 \%$ had persistent wrinkles at the root of the nose (nasal orbicular wrinkles), and 30\% had wrinkles at the nasal root and between the eyes (nasociliary wrinkles). The injection of botulinum toxin into additional locations according to the anatomic differences of each person showed excellent resolution of the rhytides without complications.

Common areas in the lower half of the face to treat with botulinum toxin are the vertical perioral rhytides. These lines are caused by repeated contraction of the orbicularis oris. Several injections of $2 \mathrm{U}$ of Botox Cosmetic can be placed along the upper and lower lip to minimize lip furrows. A study was conducted where 18 patients were injected with BTX-A into the vertical lip rhytides. The effect of treatment was evaluated at 2 to 3 weeks after procedure and smoothening of hyperfunctional lines and upper lip fullness/eversion was observed in patients treated with BTX-A injections. ${ }^{27}$

Another usage of botulinum toxin injections is to target the depressor anguli oris muscle of the mouth to elevate the corners of the mouth. ${ }^{28}$ The depressor anguli oris pulls down on the lateral oral commissures and excessive function causes the corners of the mouth to droop (marionette lines). The downward angle of the marionette lines can be improved by injecting $2-5 \mathrm{U}$ of Botox Cosmetic intramuscularly approximately $8-10 \mathrm{~mm}$ lateral to the oral commissure and $8-15 \mathrm{~mm}$ inferior to this point.

Relaxing the muscles of the mentalis can reduce the involuntary convolutions or dimpling of the chin when performing speaking or performing other mundane activities of the face. ${ }^{28}$ In addition to softening the pebbling of the chin, injection of botulinum toxin into the mentalis can reduce the deep transverse labiomental crease that runs horizontally across the chin. A dose of 5-8 U of Botox Cosmetic is typically used to inject the mentalis muscle. 
Cosmetic uses of botulinum toxin in the neck is primarily for the treatment of platysmal banding, or the vertical bands found in the central neck. ${ }^{29}$ Horizontal rhytides caused by contraction of the platysmal muscle, usually seen laterally, can be treated with botulinum toxin injections. The proper method to inject the platysma is to have the patient seated upright and contract the platysma. By doing so, the practitioner can grasp the platysmal band between the thumb and index finger with the non-dominant hand and inject 2-4 U of Botox Cosmetic in the lower dermis per injection site along the vertical extent of the band, starting approximately $2 \mathrm{~cm}$ below the inferior border of the mandible. The injections are repeated 1.5 to $2 \mathrm{~cm}$ apart from each other descending down the neck toward the border of the clavicle. Most patients will require several injection points to treat a platysmal band. It is advisable to inject no more than 40 to $50 \mathrm{U}$ of Botox Cosmetic total per treatment session for the platysmal bands.

\section{Dysport}

Dysport is used for the temporary improvement in the appearance of moderate to severe glabellar lines associated with procerus and corrugator muscle activity in adult patients less than 65 years of age.$^{30}$ The recommendation for reconstituting Dysport for glabellar lines in the $300 \mathrm{U}$ vial is with $2.5 \mathrm{~mL}$ or $1.5 \mathrm{~mL}$ of unpreserved normal saline. The recommended dosage for treating glabellar lines is a total dose of $50 \mathrm{U}$ of Dysport divided in five equal aliquots of $10 \mathrm{U}$ each to the procerus and corrugator muscles. Retreatment with Dysport at standard strength should be administered no more frequently than every 3 months. The potency units of Dysport are not interchangeable with other preparations of botulinum toxin products and, therefore, units of biological activity of Dysport cannot be compared to or converted into units of any other botulinum toxin products. ${ }^{31}$

The same anatomic areas that were described above to treat rhytides with Botox Cosmetic can also be applied with Dysport. A study was conducted to compare the efficacy and tolerability between Botox Cosmetic and Dysport in the treatment of moderate to severe glabellar lines. ${ }^{32} \mathrm{~A}$ total of 62 patients with moderate or severe glabellar lines at maximum contraction were randomly assigned to receive $20 \mathrm{U}$ of Botox Cosmetic or $50 \mathrm{U}$ of Dysport (20\% in the procerus muscle, $80 \%$ in the corrugator muscles). The incidence of patients with at least a 1-grade improvement in the severity of their glabellar lines at maximum contraction peaked at week 8 in both groups. However, the duration of this improvement was generally more prolonged with Botox
Cosmetic than with Dysport with the overall incidence of such improvement being $77 \%$ versus 59\% at week 12 and $53 \%$ versus $28 \%$ at week 16 . In addition, at week 16 , the incidence of patients whose glabellar line severity was none or mild between Botox Cosmetic and Dysport was 23\% versus $10 \%$ for all patients (not statistically significant) and $50 \%$ versus $13 \%(P=0.05)$ for patients with moderate glabellar lines at baseline. Throughout the 16-week follow-up, patients' mean scores for how attractive they felt and how satisfied they felt with their appearance were consistently higher with Botox Cosmetic than Dysport, with statistical significance achieved for both at week 12 . There was no significant between-group difference in the incidence of treatment-related adverse effects. It was shown that Botox Cosmetic offers more prolonged efficacy than Dysport when the two products were compared in a 2.5:1 dose ratio. At the 2.5:1 dose ratio, the results of the study presented herein suggest that Botox Cosmetic offers more prolonged efficacy (and comparable tolerability) relative to Dysport. The data suggest that the dose of Dysport may need to be higher than the 50-U dose suggested for the treatment of glabellar lines to achieve a duration of effect and level of patient satisfaction that is comparable with Botox Cosmetic.

The results of five phase III studies of Dysport for the treatment of glabellar lines were recently published. ${ }^{33}$ Three double-blind, multicenter, randomized, placebo-controlled studies enrolled ethnically diverse healthy adults with glabellar lines of at least moderate severity at maximum frown. In these studies, patients were followed for up to 180 days after treatment. The fixed-dose, single-treatment study randomized 158 patients to receive placebo or one dose of Dysport at $50 \mathrm{U}$. The fixed-dose, repeat-treatment study randomized 311 patients to assess treatment following the prior Dysport treatment of $50 \mathrm{U}$. The variable-dose study randomized 816 patients to receive placebo or a single variable dose (50-80 U, based on gender and assessment of muscle mass). Clinical evaluations were performed on days 14 and 30 and monthly thereafter. The primary endpoint was a response defined as a composite $2+$ grade improvement, at day 30 from baseline, on the wrinkle severity rating scale from 2 to 0 , or from 3 to 0 or 1 (where $3=$ severe wrinkles/severe, $2=$ moderate wrinkles $/$ moderate, $1=$ mild wrinkles $/$ mild , and $0=$ no wrinkles/none), for both the blinded evaluators'/blinded investigators' and patients' assessments. Patients (1,116 total; 720 Dysport, 396 placebo) given Dysport received 50 to 80 $\mathrm{U}$ of treatment. The median duration of response was 85 days for fixed dosing and 109 days for variable dosing. Similar efficacy occurred at doses adjusted for gender and muscle 
mass, although males required higher doses than females in the variable-dose study. Responses appeared as early as 24 hours, with a median time to onset of 3 days. Extension studies evaluated 1,200 patients for 13 months and 768 patients in an interim analysis for 24 months. Maintenance of efficacy was seen after multiple cycles, indicating a lack of tolerance. It was concluded that Dysport significantly improved moderate to severe glabellar lines compared with placebo, with onset seen as soon as 24 hours after treatment and a median duration of effect of 85 or 109 days for fixed or variable dosing, respectively.

In another study the efficacy of Dysport in treating crow's feet in a double-blinded placebo controlled trial was examined. ${ }^{34}$ Participants with moderate to severe crow's feet at maximum smile (mild to severe at rest) were randomized to a single bilateral Dysport treatment (15, 30 , or $45 \mathrm{U}$ ) or placebo. Independent panel assessments (Week 4) showed that all Dysport doses resulted in significant improvements in crow's feet severity at maximum smile $(P<0.001)$; a clear dose - response effect was seen. Improvement over placebo was seen in the $30-\mathrm{U}$ and 45-U groups at Week 12. Investigator assessment showed significant improvement for all doses for 12 weeks at maximum smile and rest $(P \leq 0.01)$. Patient satisfaction was significantly greater for all doses than for placebo at 16 weeks (all $P<0.05$ ).

\section{Myobloc}

Myobloc is supplied as a sterile liquid formulation of the purified neurotoxin. Each vial contains 5,000 U/mL of botulinum toxin $\mathrm{B}$ in $0.05 \%$ human serum albumin, $0.01 \mathrm{M}$ sodium succinate, and $0.1 \mathrm{M}$ sodium chloride at a slightly acidic $\mathrm{pH}$ of approximately 5.6. Myobloc is available in three different total dosage strengths: $2,500,5,000$, and 10,000 U. The recommended storage condition for Myobloc is $2^{\circ}$ to $8^{\circ} \mathrm{C}$ for up to 21 months and it must not be frozen; it can also be further diluted with normal saline. Myobloc is FDA approved for the treatment of cervical dystonia.

While not used as extensively as Botox Cosmetic or Dysport for cosmetic purposes, Myobloc may be used to treat facial rhytides in instances where patients develop loss of response to BTX-A. Repeated injections with BTX-A may result in the formation of neutralizing antibodies that may reduce the effectiveness of subsequent treatments with BTX-A by inactivating the biological activity of the toxin. However, the rate of formation of neutralizing antibodies in patients receiving BTX-A has not been well studied and the critical factors for neutralizing antibody formation have not been well characterized.
The results from some studies suggest that botulinum toxin injections at more frequent intervals or at higher doses may lead to greater incidence of antibody formation. In situations where patients develop intolerance to BTX-A, transitioning to Myobloc may lead to improved outcomes in the treatment of facial rhytides. A report demonstrated that BTX-B is an effective treatment modality for glabellar rhytides refractory or exhibiting decreased clinical efficacy to BTX-A. ${ }^{35}$

A study was published to observe the effects of BTX-B in comparison to BTX-A, on patients with brow furrows assessing initial efficacy and duration of effect. ${ }^{36}$ Patients were injected with BTX-B in two different dose conversions against BTX-A to the corrugator-procerus complex. Some patients received a conversion of $50 \mathrm{U}$ of BTX-B (a total of 1,000 U) to $1 \mathrm{U}$ of BTX-A while others received a conversion of $100 \mathrm{U}$ of BTX-B (a total of 2,000 U) to $1 \mathrm{U}$ of BTX-A. The patients treated with BTX-A received a total of $20 \mathrm{U}$. Both types of botulinum toxin were effective at improving glabellar frown lines. The onset of actions occurred slightly sooner (2-3 days) with BTX-B than with BTX-A (3-7 days). Duration of effect with BTX-A was at least 16 weeks. The duration of response was dose dependent for BTX-B with 1,000 U of lasting 6-8 weeks and the higher dose of 2,000 $U$ lasting 10-12 weeks. At least with the doses used, BTX-B has a quicker onset of action but BTX-A has longer benefit for glabellar wrinkles. These data strongly suggest that further dose ranging studies of BTX-B are necessary using controlled double blind studies in a larger patient population. In addition, there is a higher complaint of pain upon injection with Myobloc compared to Botox Cosmetic, presumably due to the lower $\mathrm{pH}$ of Myobloc. ${ }^{6,37}$

In other studies the treatment of glabellar wrinkles with BTX-B has also been investigated and a rapid onset of action but a shorter duration has been observed. A total of 1,800 U of BTX-B was used for the glabellar area and mean onset of 1.5 days and a mean duration of 8 weeks was observed. ${ }^{12} \mathrm{At}$ higher doses of BTX-B used by the same investigator in a follow-up prospective study, the duration of response was 9.6 and 10.4 weeks with 2,400 and 3,000 U, respectively. ${ }^{12}$ The onset of action was seen within 2 to 3 days. Other researchers looked at three different dosages of BTX-B for the treatment of glabellar lines. Patients received either the low dose of $1,875 \mathrm{U}$, the medium dose of 2,500 $\mathrm{U}$, or the high dose of 3,125 U.7 Most patients at all doses showed some evidence of paralysis at 2 months. At 3 months after injection, only participants in the high dose condition maintained a significant response with BTX-B. The authors concluded that at 
the doses utilized in their study, the effect of BTX-B does not generally appear to last as long as BTX-A. However, similar to the other studies, the onset of action was sooner with BTX-B.

\section{Bocouture/Xeomin}

Bocouture was approved in late 2009 in Germany for the treatment of glabellar lines in adults younger than 65 years of age. It is not available commercially in the United States. Unlike other neurotoxins, Bocouture does not contain protein complexes including bacterial proteins. Clinical trials have demonstrated that Bocouture is well tolerated and highly effective in treating the glabella lines and does not require refrigeration either during transport or in storage.

\section{Safety of botulinum toxin}

Cosmetic treatment with botulinum toxin injections has become one of the most common nonsurgical procedures performed by practitioners. It has been proven as safe and effective for the treatment of glabellar lines and other rhytides in the face and neck. Nonetheless, given its long safety track record and high usage in millions of patients, knowledge of any potential adverse effects associated with botulinum toxin is essential for any practitioner who performs such injections. Generalized reactions that have idiosyncratically occurred from botulinum toxin injections include nausea, fatigue, malaise, flu-like symptoms, and rashes at sites distant from the injection, but the instances of these events occurring is rare.

\section{Age considerations}

It is difficult to tell for sure whether patients over the age of 65 respond differently to BTX-A than do younger patients since there have been no studies investigating cosmetic uses of BTX-A specifically in the elderly, and there have not been enough elderly patients enrolled in clinical studies to make any meaningful comparisons. ${ }^{38}$ Since the elderly are more likely to have thinner and less elastic skin, weaker facial muscles, and wrinkles that over time are caused by gravity-induced tissue sagging rather than muscle contraction, the elderly are not expected to respond as well to BTX-A treatment. ${ }^{39}$ The site of injection also warrants special considerations in the elderly. Treatment of forehead lines, for example, would require injections to the frontalis muscle, which many older people use to raise their eyebrows and eyelids to see. Older patients may also have redundant skin under the brow (pseudoptosis) which could be worsened by BTX-A treatment. Older patients who receive BTX-A for glabellar lines may be more at risk for complications such as eyelid ptosis if they have a reduced or absent orbital septum. ${ }^{40}$ Conservative dosing, injection of low volumes, and proper placement of the injection can reduce the possibility of spread of the toxin to unintended muscles. A prudent method is to start at the lowest possible effective dose for elderly patients.

\section{Diffusion}

The effects of botulinum toxin effects, in some cases, may diffuse beyond the site of local injection. ${ }^{41}$ The symptoms are consistent with the mechanism of action of botulinum toxin and may include asthenia, generalized muscle weakness, diplopia, blurred vision, ptosis, and breathing difficulties. These symptoms have been reported hours to weeks after injection. To date, no definitive serious adverse event reports of distant spread of toxin effect associated with cosmetic use of botulinum toxin has been made.

\section{Pre-existing neuromuscular disorders}

Patients with peripheral motor neuropathic diseases, amyotrophic lateral sclerosis, or neuromuscular junctional disorders (myasthenia gravis or Lambert-Eaton syndrome) should be monitored particularly closely when given botulinum toxin. ${ }^{42}$ Patients with neuromuscular disorders may be at increased risk of clinically significant effects including severe dysphagia and respiratory compromise from typical doses of botulinum toxin injections.

\section{Drug interactions}

Concomitant administration of botulinum toxin and aminoglycosides or other agents interfering with neuromuscular transmission (eg, curare-like nondepolarizing blockers, lincosamides, polymyxins, quinidine, magnesium sulfate, anticholinesterases, succinylcholine chloride) should be performed with caution as the effect of the toxin may be potentiated, resulting in excessive neuromuscular weakness. ${ }^{43}$

\section{Pregnancy and lactation}

Administration of botulinum toxin is not recommended during pregnancy. There are no adequate and well-controlled studies of botulinum toxin in pregnant women. Caution should be exercised when botulinum toxin is administered to a nursing woman because it is not known whether this drug is excreted in human milk. ${ }^{43}$

\section{Ptosis and asymmetry}

Transient ptosis is the most frequently reported complication and has been reported in the literature in approximately $5 \%$ 
of patients. ${ }^{40,44}$ Ptosis of the eyelid results from migration of the botulinum toxin to the levator palpebrae superioris muscle. The levator allows the eyelid to open properly and fully. To avoid ptosis, injections should occur at least $1 \mathrm{~cm}$ above the eyebrow and should not cross the midpupillary line. A therapy recommended to treat ptosis from administration of botulinum toxins $\mathrm{A}$ and $\mathrm{B}$ is the use of apraclonidine $0.5 \%$ eye drops. The most common dosing scheme used for apraclonidine is one or two drops three times daily until ptosis resolves.

The most significant complication of treatment of the frontalis is brow ptosis. Injections in the forehead should always be above the lowest fold produced when the subject is asked to elevate their forehead (frontalis). If the patient has a low eyebrow, treatment of the forehead lines should be avoided, or limited to that portion of the forehead $4.0 \mathrm{~cm}$ or more above the brow.

The most common reported complications in the crow's feet area are bruising, diplopia, ectropion, and an asymmetric smile due to injection of the zygomaticus major. ${ }^{40}$ If severe lower lid weakness occurs, an exposure keratitis may result. Treatment is symptomatic. These complications are avoided by injecting at least $1 \mathrm{~cm}$ outside the bony orbit or $1.5 \mathrm{~cm}$ lateral to the lateral canthus, not injecting medial to a vertical line through the lateral canthus, and not injecting close to the inferior margin of the zygoma. Violating these boundaries has on occasion also resulted in diplopia due to medial migration of Botox and resultant paralysis of the lateral rectus muscle. Covering or patching the eye will alleviate some of the double vision.

\section{Patient reported outcomes with botulinum toxin}

In the field of cosmetic therapy, patient satisfaction is vital and patient perceptions of the success of treatment are influenced both by their expectations before treatment and by their impressions of the clinical outcome. Patients' perceptions of clinical outcome are complex, and patient evaluations that have previously been reported in clinical trials for glabellar lines have typically been broad-based general assessments, such as the global assessment of change in the appearance of glabellar lines or the general level of satisfaction with treatment. Although these offer valuable insights into the patients' overall perceptions, they may not address specific aspects of treatment that are of greatest importance to an individual patient - for example, some patients want to look younger, some want to be more attractive, some would like to feel better about their appearance, some want to look more rested, and some want to avoid looking tired, stressed, or angry when this is not how they feel. To help evaluate the success of treatment in achieving individual goals such as these, it is necessary to use more detailed assessments of patientreported outcomes that evaluate these issues specifically.

Several patient-reported outcomes measures have been developed and utilized in clinical trials of botulinum toxin for the treatment of rhytides, including the Self-Perception of Age (SPA) measure and the Facial Line Outcomes (FLO) questionnaire. On the FLO questionnaire, patients rate the degree to which their facial lines bother them; make them look older than they would like; prevent them from having a smooth facial appearance; and make them look tired, stressed, or angry when that is not how they feel. In several clinical trials significant improvements in these outcomes have been demonstrated from baseline and versus placebo for the treatment of multiple upper facial lines as well as for treating the glabella as a single region. These outcomes data help in understanding patient objectives and motivations, establishing a treatment plan, and ensuring patient satisfaction. ${ }^{45}$

In one study it was demonstrated that Botulinum toxin type A showed significantly superior efficacy to placebo in terms of both glabellar line severity (at maximum contraction and at rest) and global assessment. ${ }^{46}$ The mean score from the FLO questionnaire was also significantly greater with botulinum toxin type A than placebo, indicating that patients' perceptions of their appearance had been significantly improved by botulinum toxin. This was reflected in the self-perception of age data, which showed that botulinum toxin resulted in more patients considering themselves to look younger than their current age and fewer patients considering themselves to look older than their current age. Furthermore, those who considered that they looked younger than their current age at Week 4 but not at baseline reported a large change in the perception of their age (from a median of 4 years older than their current age at baseline to 5 years younger than their current age at Week 4).

In this study it was demonstrated that botulinum toxin type A offers not only clinical efficacy but also efficacy in addressing aspects of treatment that are of particular importance to patients - such as their desire to improve their facial appearance, have a smoothness to their facial appearance, not look older than they want, and not look tired, stressed, and angry when this is not how they feel. As patient perceptions such as these are actually the greatest determinant of treatment success in everyday clinical practice than more objective evaluations of clinical improvement, it is reassuring to know that botulinum toxin type A delivers what patients most desire. Botulinum toxin type A can help 
patients achieve specific goals of treatment, such as those described above.

In another study, the objective was to compare the effect of Botox Cosmetic with placebo on participant-reported outcomes and to assess the utility of $64 \mathrm{U}$ of Botox Cosmetic to treat the entire upper face. ${ }^{47} \mathrm{~A}$ total of 40 female participants were randomized to receive $64 \mathrm{U}$ of Botox Cosmetic or identical placebo injections (double-masked) divided among 16 sites of the upper face and were followed for 12 weeks. Participants unimproved at Week 4 were eligible for open-label Botox Cosmetic treatment and were followed through Week 16. Main outcome measures were scores on seven items of the FLO questionnaire and results on the SPA questionnaire for assessing age of appearance relative to actual age. Botulinum toxin treatment increased the proportion of participants who reported looking younger than their current age while placebo injections had no effect on age perception. Throughout the duration of the study, 75\% of the botulinum toxin-treated patients reported looking younger. At 4 weeks after treatment, botulinum toxin-treated participants reported looking younger by 3 years compared with the perceived age at baseline.

In another study it was demonstrated that BTX-A treatment for frown lines can also be used as a treatment for depression. ${ }^{48}$ A psychological mechanism for this effect is that relaxation of the frown muscles leads to less facial feedback for negative emotions. Consequently, a negative affect is harder to maintain and so the person has a more positive mood. In order to test this mechanism, the mood of patients who had received BTX-A treatment for glabellar frown lines was measured and compared with patients who had received other cosmetic treatments. The results showed that the BTX-A-treated patients showed a significantly less negative mood and supported the facial feedback view that frowning can make one unhappier. Treatments that prevent frowning correlate with reduced negative mood.

\section{Conclusion}

The aforementioned therapeutic uses of the various types of botulinum toxin formulations have expanded the ability of practitioners to treat various rhytides of the face and neck and to reverse the signs of aging. By specially targeting the hyperkinetic lines of the face, botulinum toxin has addressed a fundamental problem of recurring wrinkles that could not be adequately controlled with filler agents or laser treatment by themselves. When used as monotherapy or in combination with other treatment modalities, the use of botulinum toxin is a simple, minimally-invasive procedure that has minimal to no downtime, minimal adverse events, and has turned back the clock for millions of patients.

\section{Disclosure}

The author reports no conflicts of interest.

\section{References}

1. Petrus GM, Lewis D, Maas CS. Anatomic considerations for treatment with botulinum toxin. Facial Plast Surg Clin North Am. 2007;1:1-9.

2. Ward AB, Molenaers G, Colosimo C, Berardelli A. Clinical value of botulinum toxin in neurological indications. Eur J Neurol. 2006;4: 20-26.

3. Carruthers JDA and Carruther JA. Treatment of glabellar frown lines with C. botulinum A exotoxin. J Dermatol Surg Oncol. 1991;18:17-21.

4. Lowe NJ, Ascher B, Heckmann M, Kumar C, Fraczek S, Eadie N. Botox Facial Aesthetics Study Team. Double-blind, randomized, placebo-controlled, dose-response study of the safety and efficacy of botulinum toxin type A in subjects with crow's feet. Dermatol Surg. 2005;31(3):257-262.

5. Carruthers A, Carruthers J. A single-center, dose-comparison, pilot study of botulinum neurotoxin type A in female patients with upper facial rhytids: safety and efficacy. J Am Acad Dermatol. 2009;60(6): 972-979.

6. Lowe NJ, Yamauchi PS, Lask GP, Patnaik R, Moore D. Botulinum toxins types A and B for brow furrows: preliminary experiences with type B toxin dosing. J Cosmet Laser Ther. 2002;4(1):15-18.

7. Spencer JM, Gordon M, Goldberg DJ. Botulinum B treatment of the glabellar and frontalis regions: a dose response analysis. J Cosmet Laser Ther. 2002;4(1):19-23.

8. Sadick NS, Faacs. Botulinum toxin type B for glabellar wrinkles: a prospective open-label response study. Dermatol Surg. 2002;28(9): 817-821.

9. Matarasso SL. Comparison of botulinum toxin types A and B: a bilateral and double-blind randomized evaluation in the treatment of canthal rhytides. Dermatol Surg. 2003;29(1):7-13.

10. Sadick NS, Herman AR. Comparison of botulinum toxins A and B in the aesthetic treatment of facial rhytides. Dermatol Surg. 2003;29(4):340-347.

11. Sadick NS. Botulinum toxin type B. Dermatol Surg. 2003;29(4): 348-350.

12. Sadick NS. Prospective open-label study of botulinum toxin type B (Myobloc) at doses of 2,400 and 3,000 U for the treatment of glabellar wrinkles. Dermatol Surg. 2003;29(5):501-507.

13. Baumann L, Slezinger A, Vujevich J, Halem M, Bryde J, Black L, Duncan R. A double-blinded, randomized, placebo-controlled pilot study of the safety and efficacy of Myobloc (botulinum toxin type B)-purified neurotoxin complex for the treatment of crow's feet: a double-blinded, placebo-controlled trial. Dermatol Surg. 2003;29(5):508-515.

14. Schantz EJ. Historical perspective. In: Jankovic J, Hallet M, editors. Therapy with botulinum toxin. New York: Marcel Dekker, Inc; 1994: xxiii-xxvi.

15. Coffield JA, Considine RV, Simpson LL. The site and mechanism of action of botulinum neurotoxin. In: Jankovic J, Hallet M, editors. Therapy with botulinum toxin. New York: Marcel Dekker, Inc; 1994:3-13.

16. Martin TFJ. Stages of regulated exocytosis. Trends Cell Biology. 1997; 7:271-276.

17. Blasi J, Chapman ER, Link E, et al. Botulinum neurotoxin A selectively cleaves the synaptic protein SNAP-25. Nature. 1993;365(6442): 160-163.

18. Schiavo G, Benfenati F, Poulain B, et al. Tetanus and botulinum-B neurotoxins block neurotransmitter release by proteolytic cleavage of synaptobrevin. Nature. 1992;359:832-835.

19. Alam M, Dover JS, Arndt KA. Pain associated with injection of botulinum A exotoxin reconstituted using isotonic sodium chloride with and without preservative: a double-blind, randomized controlled trial. Arch Dermatol. 2002;138(4):510-514. 
20. Yomtoob DE, Dewan MA, Lee MS, Harrison AR. Comparison of pain scores with 30-gauge and 32-gauge needles for periocular botulinum toxin type a injections. Ophthal Plast Reconstr Surg. 2009; 25(5):376-377.

21. Frankel AS, Markarian A. Cosmetic treatments and strategies for the upper face. Facial Plast Surg Clin North Am. 2007;15(1):31-39.

22. Carruthers JA, Lowe NJ, Menter MA, et al. Botox Glabellar Lines I Study Group. A multicenter, double-blind, randomized, placebo-controlled study of the efficacy and safety of botulinum toxin type A in the treatment of glabellar lines. J Am Acad Dermatol. 2002;46(6):840-849.

23. Maas CS, Kim EJ. Temporal brow lift using botulinum toxin A: an update. Plast Reconstr Surg. 2003;112:S109-S112.

24. Redaelli A, Forte R. How to avoid brow ptosis after forehead treatment with botulinum toxin. J Cosmet Laser Ther. 2003;5(3-4):220-222.

25. Lowe NJ, Shah A, Lowe Pl, Patnaik R. Dosing, efficacy and safety plus the use of computerized photography for botulinum toxins type A for upper facial lines. J Cosmet Laser Ther. 2010;12(2):106-111.

26. Tamura BM, Odo MY, Chang B, Cucé LC, Flynn TC. Treatment of nasal wrinkles with botulinum toxin. Dermatol Surg. 2005;31(3):271-275.

27. Semchyshyn N, Sengelmann RD. Botulinum toxin A treatment of perioral rhytides. Dermatol Surg. 2003;29(5):490-495.

28. Flynn TC. Update on botulinum toxin. Semin Cutan Med Surg. 2006; 25(3):115-121.

29. Matarasso A, Matarasso SL. Botulinum A exotoxin for the management of platysma bands. Plast Reconstr Surg. 2003;112:S138-S140.

30. Hexsel D, Spencer JM, Woolery-Lloyd H, Gilbert E. Practical applications of a new botulinum toxin. J Drugs Dermatol. 2010;9:S31-S37.

31. Wohlfarth K, Sycha T, Ranoux D, Naver H, Caird D. Dose equivalence of two commercial preparations of botulinum neurotoxin type A: time for a reassessment? Curr Med Res Opin. 2009;25(7):1573-1584.

32. Lowe P, Patnaik R, Lowe N. Comparison of two formulations of botulinum toxin type A for the treatment of glabellar lines: a double-blind, randomized study. J Am Acad Dermatol. 2006;55(6):975-980.

33. Baumann L, Brandt F, Kane M. An analysis of efficacy data from five phase III studies of BoNT-A for the treatment of glabellar lines. J Am Acad Dermatol. 2010;62(3):AB40.

34. Ascher B, Rzany BJ, Grover R. Efficacy and safety of botulinum toxin type A in the treatment of lateral crow's feet: double-blind, placebo-controlled, dose-ranging study. Dermatol Surg. 2009;35(10): $1478-1486$.
35. Alster TS, Lupton JR. Botulinum toxin type B for dynamic glabellar rhytides refractory to botulinum toxin type A. Dermatol Surg. 2003;29(5):516-518.

36. Yamauchi PS, Lowe NJ. Botulinum toxin types A and B: comparison of efficacy, duration, and dose-ranging studies for the treatment of facial rhytides and hyperhidrosis. Clin Dermatol. 2004;22(1):34-39.

37. Carruthers JA, Lowe NJ, Menter MA, et al. Botox Glabellar Lines I Study Group. A multicenter, double-blind, randomized, placebo-controlled study of the efficacy and safety of botulinum toxin type A in the treatment of glabellar lines. J Am Acad Dermatol. 2002;46(6):840-849.

38. Cheng CM. Cosmetic use of botulinum toxin type A in the elderly. Clin Interv Aging. 2007;2(1):81-83.

39. Rhodes LM, Norman RH, Wrone DA, et al. Cutaneous surgery in the elderly: ensuring comfort and safety. Dermatol Ther. 2003; $16: 243-253$.

40. Carruthers J, Fagien S, Matarasso SL, et al. Consensus recommendations on the use of botulinum toxin type A in facial aesthetics. Plast Reconstr Surg. 2004;114:S1-S22.

41. de Almeida AT, De Boulle K. Diffusion characteristics of botulinum neurotoxin products and their clinical significance in cosmetic applications. J Cosmet Laser Ther. 2007;9:17-22.

42. Borodic G. Myasthenic crisis after botulinum toxin. Lancet. 1998; 352(9143): 1832 .

43. Schaffner R, Kreyden OP. Complications and side-effects of botulinum toxin A. Curr Probl Dermatol. 2002;30:141-148.

44. Klein AW. Complications with the use of botulinum toxin. Dermatol Clin. 2004;22(2):197-205.

45. Carruthers A, Carruthers J. Patient-reported outcomes with botulinum neurotoxin type A. J Cosmet Laser Ther. 2007;9:32-37.

46. Fagien S, Cox SE, Finn JC, Werschler WP, Kowalski JW. Patient-reported outcomes with botulinum toxin type A treatment of glabellar rhytids: a double-blind, randomized, placebo-controlled study. Dermatol Surg. 2007;33:S2-S9.

47. Carruthers J, Carruthers A. Botulinum toxin type A treatment of multiple upper facial sites: patient-reported outcomes. Dermatol Surg. 2007;33:S10-S17.

48. Lewis MB, Bowler PJ. Botulinum toxin cosmetic therapy correlates with a more positive mood. J Cosmet Dermatol. 2009;8(1):24-26.
Patient Preference and Adherence

\section{Publish your work in this journal}

Patient Preference and Adherence is an international, peer-reviewed, open access journal focusing on the growing importance of patient preference and adherence throughout the therapeutic continuum. Patient satisfaction, acceptability, quality of life, compliance, persistence and their role in developing new therapeutic modalities and compounds to

\section{Dovepress}

optimize clinical outcomes for existing disease states are major areas of interest. This journal has been accepted for indexing on PubMed Central. The manuscript management system is completely online and includes a very quick and fair peer-review system. Visit http://www.dovepress.com/ testimonials.php to read real quotes from published authors. 